\title{
Habilidades Sociais Profissionais e Indicadores de Ansiedade e Depressão em Gestores
}

\author{
Edward Goulart Júnior ${ }^{1}$ \\ ${ }^{1}$ Universidade Estadual Paulista Júlio de Mesquita Filho, \\ Bauru, SP, Brasil. \\ Hugo Ferrari Cardoso ${ }^{1}$ \\ ${ }^{1}$ Universidade Estadual Paulista Júlio de Mesquita Filho, \\ Bauru, SP, Brasil. \\ Thaís Almeida Alves ${ }^{1}$ \\ ${ }^{1}$ Universidade Estadual Paulista Júlio de Mesquita Filho, \\ Bauru, SP, Brasil. \\ Aline de Marco da Silveira ${ }^{1}$ \\ ${ }^{1}$ Universidade Estadual Paulista Júlio de Mesquita Filho, \\ Bauru, SP, Brasil.
}

Resumo: A busca por profissionais comprometidos com o trabalho e que saibam estabelecer relações pessoais saudáveis e produtivas é o grande desafio atual dos setores de gestão de pessoas, que precisam investir cada vez mais em desenvolvimento humano e na qualidade das interações profissionais. Uma temática de análise que tem ganhado destaque nesse cenário é a de habilidades sociais profissionais. O presente estudo avaliou o repertório de habilidades sociais profissionais manifestadas nos comportamentos de 43 gestores atuantes em setores administrativos de uma Instituição de Ensino Superior (IES) Pública, assim como os indicadores de depressão e ansiedade e possíveis associações entre os construtos. Foram aplicados os instrumentos: Inventário de Habilidades Sociais Profissionais de Gestores (IHSP-G), Inventário de Ansiedade de Beck (BAI) e Inventário de Depressão de Beck (BDI). Os resultados demonstraram que as categorias do IHSP-G com menores médias entre os gestores foram: Cumprimentos de Metas e Objetivos Profissionais, Gestão de Pessoas e Resoluções de Conflitos no Trabalho. Nos dados do BAI a pontuação média indicou ansiedade moderada, enquanto no BDI constatou-se a ausência de indicadores de depressão. Foram encontradas correlações negativas entre as categorias do IHSP-G e os instrumentos BAI e BDI. Pode-se formular a hipótese de que gestores habilidosos apresentam menores indicadores de depressão e ansiedade.

Palavras-chave: Habilidades Sociais, Habilidades Sociais Profissionais, Ansiedade, Depressão.

\section{Professional Social Skills and Indicators of Anxiety and Depression in Managers}

\begin{abstract}
The quest for professionals committed to work and able to establish healthy and productive personal relationships is the greatest modern challenge to People Management, spurring increasing investments in human development and quality professional interactions. In this scenario, professional social skills is a subject of analysis that has gained prominence. This research evaluated the repertoire of professional social skills in 43 managers in the administrative sectors of a public higher education institution, as well as indicators of depression and anxiety and possible associations between these constructs. For that, the following instruments were used: Management Professional Social Skills Inventory (MPSSI), Beck Anxiety Inventory (BAI), and Beck Depression Inventory (BDI). The results show that managers reached the lowest means in accomplishment of professional aims and objectives, people management, and resolution of conflicts at work. The mean score at the BAI indicates moderate anxiety and, and BDI suggests the absence of depression indicators. Negative correlations between MPSSI categories and both BAI and BDI were found. Thus, this study hypothesis is that skilled managers have lower depression and anxiety indicators.
\end{abstract}

Keywords: Social Skills, Professional Social Skills, Anxiety, Depression. 


\title{
Habilidades Sociales Profesionales e Indicadores de Ansiedad y Depresión en Gestores
}

\begin{abstract}
Resumen: La búsqueda de profesionales comprometidos con el trabajo y que sepan establecer relaciones personales saludables y productivas constituye el gran desafío actual de los sectores de gestión de personas, que necesita inversiones cada vez más en el desarrollo humano y en la calidad de las interacciones profesionales. Un tema de análisis que ha ganado destaque en este escenario es el de habilidades sociales profesionales. Este estudio evaluó el repertorio de habilidades sociales profesionales presentes en los comportamientos de 43 gestores que trabajan en sectores administrativos de una Institución de Enseñanza Superior (IES) pública, así como los indicadores de depresión y ansiedad, con las posibles asociaciones entre los constructos. Se aplicaron los siguientes instrumentos: Inventario de Habilidades Sociales Profesionales de Gestores (IHSP-G), Inventario de Ansiedad de Beck (BAI) y el Inventario de Depresión de Beck (BDI). Los resultados demostraron que el cumplimiento de metas y objetivos profesionales, la gestión de personas y las resoluciones de conflictos en el trabajo fueron las categorías del IHSP-G que presentaron las menores medias entre los gestores. En los datos del BAI el promedio de puntaje indicó ansiedad moderada, mientras que en el BDI apuntó ausencia de indicadores de depresión. Se encontraron correlaciones negativas entre las categorías del ISHP-G y los instrumentos BAI y BDI. Se puede hipotetizar que los gestores habilidosos presentan menores indicadores de depresión y ansiedad.
\end{abstract}

Palabras clave: Habilidades Sociales, Habilidades Sociales Profesionales, Ansiedad, Depresión.

\section{Introdução}

O mundo do trabalho contemporâneo caracteriza-se por profundas e constantes mudanças de diversos âmbitos: econômicos, sociais, comunicacionais, políticos etc. Esse cenário predispõe as organizações a atuarem em um contexto de instabilidade e de crescente competitividade, e elas, muitas vezes, precisam responder rapidamente e com eficiência às novas demandas que se apresentem (Portz \& Amazarray, 2019; Rodrigues \& Faiad, 2019). Urge a pertinência de refletir acerca de políticas de gestão por meio das quais torne-se possível planejar e gerir de forma adequada tanto as pessoas que compõem a organização como os produtos por ela oferecidos ou prestados (Zanelli \& Silva, 2008).

A busca por profissionais comprometidos com o trabalho e que saibam estabelecer relações pessoais saudáveis e produtivas, é o grande desafio atual dos setores de gestão de pessoas, que precisam investir cada vez mais em desenvolvimento humano e na qualidade das interações profissionais. Uma temática de análise que tem ganhado destaque nesse cenário, com maior ênfase na literatura internacional do que na nacional, é a de habilidades sociais profissionais (Silveira, Goulart Júnior, Cardoso, \& Bolsoni-Silva, 2016). Com base em Argyle (1980), habilidades sociais profissionais são um conjunto de repertórios comportamentais complexos que possibilita ao indivíduo interagir profissionalmente de forma a atender as demandas de trabalho por meio de relações interpessoais satisfatórias e eficientes, visando ao cumprimento de objetivos profissionais, bem como à presença de um ambiente saudável e produtivo para si e para os outros, em respeito aos direitos e às diferenças individuais.

Rodrigues, Araújo, Ribeiro e Rubio (2015) apontam que os estudos em habilidades sociais profissionais auxiliam as organizações e os trabalhadores, na medida em que aumentam a eficácia individual e grupal e, consequentemente, otimizam os resultados organizacionais. O bom repertório em habilidades sociais é tido como um fator protetivo em relação ao desenvolvimento de morbidades psicológicas como a depressão e ansiedade, além de estar relacionado à sensação de bem-estar, a melhores resultados profissionais e ao desejo do trabalhador de continuar em seu emprego (Segrin \& Rynes, 2009; Segrin \& Taylor, 2007). 
Entende-se, portanto, que a busca por um ambiente de trabalho que promova a saúde e qualidade de vida dos trabalhadores exige o desenvolvimento de habilidades sociais profissionais, principalmente para os profissionais que exercem cargos de gestão. Assim, o objetivo deste artigo é apresentar estudo que visou avaliar, junto a gestores que atuam em setores técnico-administrativos de uma IES Pública, o repertório de habilidades sociais profissionais manifestados em seus comportamentos, bem como mensurar seus indicadores de depressão e ansiedade. De forma adicional, a pesquisa também visou verificar as associações entre habilidades sociais profissionais e indicadores de depressão e ansiedade. Desenvolver estudos sobre as habilidades sociais profissionais pode trazer importantes contribuições não somente para ações de formação, capacitação e desenvolvimento de gestores, mas também para a manutenção de um ambiente organizacional promotor de saúde e satisfação.

\section{Habilidades sociais: conceitos, sua importância no âmbito laboral e relações com indicadores de saúde mental}

Há diversas definições acerca das habilidades sociais, como a defendida por Argyle, Furnaham e Graham (1981), que conceituam habilidades sociais como um conjunto de desempenhos que um indivíduo apresenta diante de uma situação interpessoal. Para Caballo (2003) e Falcone (2002), as habilidades sociais são os comportamentos mais requeridos ao sucesso do estabelecimento de uma interação interpessoal e incluem comportamentos como: iniciar, manter e finalizar conversas; fazer e responder perguntas; pedir ajuda; recusar pedidos; e escutar de forma empática.

O conceito apresentado por Del Prette e Del Prette (2001) é amplamente aceito por estudiosos da área e classifica o comportamento socialmente habilidoso em seis categorias: habilidades sociais de comunicação; de civilidade; assertivas de enfrentamento; empáticas; habilidades sociais de trabalho; e habilidades sociais de expressão de sentimento positivo. No que tange ao trabalho, ele abrange comportamentos importantes para o ambiente profissional, como a capacidade de coordenar grupos e resolver problemas.

Os estudos sobre habilidades sociais no ambiente de trabalho, aqui chamadas de habilidades sociais profissionais, são escassos, principalmente no Brasil. Em acréscimo, a produção nacional em habilidades sociais tem sido investigada em maior escala em outros públicos ou no âmbito de características específicas de não trabalhadores.

Ao se referirem mais especificamente ao contexto de trabalho, alguns autores (Cerutti \& Wagner, 2014; Cunha \& Conceição, 2013) usam o termo "Habilidades Sociais Profissionais", criado por Argyle (1980), que distingue essas habilidades como aquelas que atendem às diferentes demandas interpessoais do ambiente de trabalho objetivando o cumprimento de metas, a preservação do bem-estar da equipe e o respeito aos direitos de cada indivíduo. Foram encontrados dois estudos brasileiros mais específicos sobre habilidades sociais no contexto de trabalho. O primeiro é de Cunha e Conceição (2013), que avaliou o repertório de habilidades sociais de militares; a segunda é a pesquisa de Cerutti e Wagner (2014), que investigou o repertório de habilidades sociais em funcionários de uma IES que trabalhavam diretamente com atendimento ao público. Entretanto, ambos utilizaram o Inventário de Habilidades Sociais (IHS), e não um instrumento direcionado para a mensuração de habilidades sociais profissionais.

Partindo do princípio de que as habilidades profissionais estão relacionadas às sociais de forma geral, no que diz respeito à relação entre habilidades sociais e saúde, as pesquisas empíricas apontam que possuir repertório social habilidoso seria um fator de proteção contra morbidades psicológicas (Campos, Del-Prette, \& Del-Prette, 2014). Estudos indicam que o déficit de comportamentos socialmente habilidosos pode ser considerado como um fator de risco no desenvolvimento de depressão e de ansiedade (Bolsoni-Silva \& Loureiro, 2016; Campos, Del-Prette, \& Del-Prette, 2018; Segrin \& Rynes, 2009). Del-Prette e Del-Prette (2002) afirmaram que os transtornos afetivos e a ansiedade podem causar perturbações nas relações sociais e que sintomas depressivos estão relacionados com dificuldades em competência social.

Espera-se que nos contextos de trabalho as interações sejam estabelecidas de forma habilidosa, tanto para o atingimento de objetivos como para o favorecimento de um clima profissional adequado, promotor de saúde e bem-estar (Grubaugh \& Flynn, 2018; Hoffman \& Tadelis, 2021). Nesse sentido, os gestores recebem atenção de destaque, na medida em que suas interações são intensas e complexas e possuem papel crucial no clima de trabalho e nos resultados organizacionais (Bryan et al., 2018; Gayed et al., 2018). 
Muito se exige desses profissionais quanto a sua capacidade de estabelecer relacionamentos que promovam comprometimento e satisfação individual e grupal. Entretanto, devido à complexidade da atuação dos gestores, há estudos que apontaram para o fato de que os indicadores de depressão e ansiedade são considerados elevados e prejudiciais à saúde desses profissionais (Hentrich, Zimber, Sosnowsky-Waschek, Gregersen, \& Petermann, 2017; Lourenção, 2018; Pires et al., 2018).

Goulart Júnior, Camargo e Moreira (2019) realizaram um estudo de análise da literatura científica sobre habilidades sociais profissionais nas bases de dados Scielo, PePSICo, BVS-Psi, Lilacs, Latindex e Redalyc, em que foram localizados e analisados 38 artigos científicos. No que tange à avaliação de habilidades sociais profissionais de gestores, apontaram que a literatura nacional é bastante escassa, de modo que não foi possível localizar, por exemplo, um instrumento (construído por pesquisadores brasileiros ou mesmo adaptado) que mensure especificamente as habilidades sociais profissionais nesse grupo. Também não foram encontrados artigos que tivessem como propósito a avaliação de habilidades sociais profissionais de gestores e a relação delas com ansiedade ou mesmo depressão.

Considerando a escassa produção que relaciona habilidades sociais profissionais, o desempenho profissional de gestores e a saúde mental, o presente estudo vem dar sua contribuição para o avanço e favorecimento de novas investigações sobre habilidades sociais profissionais nos âmbitos organizacionais, bem como sobre seus fatores antecedentes e consequentes.

\section{Método}

\section{Participantes}

Participaram do estudo 43 gestores técnicos administrativos atuantes em Unidades Acadêmicas e Administrativas de uma Instituição de Ensino Superior (IES) pública. Dentre eles, 6 declararam ocupar um cargo de gerência intermediária, 4 de supervisão operacional, 22 de supervisão administrativa, 3 de outras formas de gerência e 8 não responderam ao item.

Ao todo, 17 lideranças se identificaram como pertencentes ao gênero masculino, 20 ao feminino e 6 não responderam ao item. As idades do grupo amostral variaram entre 27 e 55 anos e a quase totalidade (42) possuía ensino superior completo. Em relação à atuação profissional, o tempo de emprego na IES variou de dois a 39 anos e o de atividade no cargo de gestão, entre cinco meses e 17 anos. Os participantes possuíam uma média de 13 profissionais sob sua coordenação e uma carga horária laboral média de 35 horas semanais.

\section{Materiais}

\section{Inventário de Habilidades Sociais Profissionais de Gestores (IHSP-G)}

Tem como objetivo avaliar o repertório de habilidades sociais de profissionais que exercem cargo de gestão com base em oito categorias, quais sejam: Relacionamento interpessoal, Cumprimento de metas e objetivos profissionais, Respeito aos direitos e diferenças individuais, Comunicação organizacional, Resoluções de conflitos no trabalho, Gestão do desempenho humano no trabalho, Gestão de pessoas e a categoria Lidar com o estresse e estressores profissionais. É composto por 89 itens (situações que podem ocorrer no contexto laboral desse gestor), respondidos por meio de uma escala do tipo Likert de cinco pontos (Sempre; Frequentemente; Às vezes; Nunca; e Não se aplica).

O IHSP-G foi construído levando-se em consideração as categorias apresentadas na literatura sobre habilidades sociais profissionais e as competências esperadas para cargos de gestão (Argyle, 1980; Bolsoni-Silva \& Loureiro, 2016; Del Prette \& Del Prette, 2001; Rodrigues et al., 2015; Silveira et al., 2016), uma vez que não foi identificado outro instrumento construído ou adaptado para o contexto brasileiro que avaliasse o referido construto em gestores. Atualmente, um instrumento dessa natureza está em fase de elaboração para que possam ser realizados futuramente estudos psicométricos, especialmente evidências de validade com base na estrutura interna e de confiabilidade (Goulart Júnior, Cardoso, Bolsoni-Silva, \& Silveira, 2017).

\section{Escalas Beck para a avaliação da ansiedade e depressão}

Adotou-se as escalas Beck (Cunha, 2001) para a mensuração dos indicadores de ansiedade e depressão, uma vez que foram os instrumentos utilizados em alguns dos estudos recuperados que tiveram 
gestores como participantes (Lourenção, 2018; Pires et al., 2018). De forma mais específica, o Inventário de Ansiedade de Beck (BAI) avalia a presença de sintomas de ansiedade em pessoas com mais de 17 anos. É composto por 21 itens com afirmações descritivas de ansiedade que devem ser avaliadas pelo respondente em referência a si mesmo a partir de uma escala de 4 pontos que apresenta níveis de gravidade crescentes a cada sintoma. $\mathrm{O}$ escore resulta na classificação em níveis de intensidade da ansiedade, que pode ser sintomatologia mínima, leve, moderada e grave.

O Inventário de Depressão de Beck (BDI) avalia a presença de sintomas depressivos em pessoas com idade entre 17 e 80 anos. É formado por 21 itens, cada um dos quais apresenta quatro alternativas com graus crescentes de gravidade de depressão. O escore total possibilita a classificação em níveis de intensidade de depressão, os quais dizem respeito a sintomatologia mínima, leve, moderada e grave.

\section{Procedimento de coleta de dados}

Antes da aplicação dos instrumentos nos participantes, o projeto de pesquisa foi submetido e aprovado pelo Comitê de Ética em Pesquisa (CAAE: 45439215.1.0000.5398). A coleta de dados foi realizada prioritariamente em grupos, em local e horários previamente programados. Os gestores que não puderam comparecer foram convidados a participar de aplicações individuais. Foi apresentado para cada participante um Termo de Consentimento Livre e Esclarecido (TCLE), que foi lido e assinado de imediato, e os três instrumentos descritos no tópico anterior (IHSP-G, BAI e BDI). Houve explicações sobre a relevância do projeto, o sigilo da pesquisa e, por fim, as instruções para a realização da coleta.

\section{Análise de dados}

Os dados foram analisados de forma descritiva e inferencial. Nas análises descritivas foram verificadas as médias de pontuações entre os instrumentos aplicados, com a finalidade de verificar as classificações obtidas pelos sujeitos de acordo com as normas contidas nos manuais dos instrumentos utilizados. As análises inferenciais foram feitas por meio da correlação de Pearson e tiveram o objetivo de verificar a possível associação entre o repertório de habilidades sociais profissionais dos gestores, os indicadores de ansiedade e os indicadores de depressão.

\section{Resultados e discussões}

A apresentação dos resultados será feita em duas partes: na primeira, expõe-se os dados descritivos obtidos por intermédio das médias das respostas dos gestores nos três instrumentos aplicados (IHSP-G, BAI e BDI); depois, serão apresentadas as correlações entre as categorias do IHSP-G e os instrumentos BAI e BDI.

$\mathrm{O}$ interesse em investigar os gestores decorreu do fato de eles assumirem diversas responsabilidades para com a organização e a equipe de trabalho que lidera, ações que requerem conhecimentos e comportamento habilidoso por parte deles. Tem-se como hipótese que o baixo repertório de habilidades sociais profissionais pode ser um dos desencadeadores do adoecimento em termos de saúde mental em gestores. Para Grubaugh e Flynn (2018) e Hoffman e Tadelis (2021), o gestor deve superar o papel de tomador de decisões, transformando-se em educador, facilitador, empreendedor, professor e guia. Os autores destacam ainda que a presença das habilidades sociais profissionais no repertório comportamental de trabalhadores torna-se quase uma exigência para o êxito profissional, uma vez que há o reconhecimento de que, para a obtenção de resultados organizacionais satisfatórios, necessita-se de relações positivas e de qualidade entre seus membros (Rodrigues et al., 2015).

Silveira et al. (2016) ressaltam que a literatura nacional sobre habilidades sociais em contextos de trabalho, embora seja um tema em ascensão, limita-se quase exclusivamente a descrever treinamentos oferecidos pelas organizações de trabalho, com poucas tentativas de identificação de repertórios socialmente habilidosos presentes nas amostras pesquisadas. Com o intuito de mensurar o repertório de habilidades sociais profissionais de gestores, Goulart Júnior et al. (2017) construíram o IHSP-G. No referido instrumento, como já descrito, as pontuações obtidas podiam variar entre 0 (Não se aplica) e 4 (Sempre), de forma que quanto mais próximo ao 4 , maior é o repertório de habilidades sociais profissionais do respondente. Dessa forma, para a interpretação dos dados neste estudo, pontuações entre 1 e 2,99 foram consideradas abaixo da pontuação mediana da frequência de comportamentos habilidosos apresentados pelos gestores; e pontuações entre 3 e 4 dizem respeito à presença de mais indicadores de comportamentos habilidosos. A Tabela 1 apresenta os valores médios obtidos dos gestores em cada categoria mensurada. 
Tabela 1

Categorias de habilidades sociais profissionais para gestores.

\begin{tabular}{lc}
\hline \multicolumn{1}{c}{ CATEGORIA } & MÉDIA \\
\hline Relacionamento interpessoal & 3,11 \\
$\begin{array}{l}\text { Cumprimento de metas e objetivos } \\
\text { profissionais }\end{array}$ & 2,99 \\
$\begin{array}{l}\text { Respeito aos direitos e diferenças } \\
\text { individuais }\end{array}$ & 3,41 \\
$\begin{array}{l}\text { Comunicação organizacional } \\
\text { Resoluções de conflitos no trabalho }\end{array}$ & 3,11 \\
$\begin{array}{l}\text { Gestão do desempenho humano no } \\
\text { trabalho }\end{array}$ & 3,07 \\
$\begin{array}{l}\text { Gestão de pessoas } \\
\text { Lidar com o estresse e estressores } \\
\text { profissionais }\end{array}$ & 2,60 \\
\hline
\end{tabular}

A partir do crivo adotado pelo presente estudo, constatou-se que em todas as categorias (com exceção das Cumprimento de metas e objetivos profissionais e Gestão de pessoas) os participantes apresentaram pontuações médias acima de 3 , o que indica elevada presença de comportamentos habilidosos. A seguir, os resultados de cada categoria serão apresentados e discutidos com base em constatações de outros estudos.

A primeira categoria do IHSP-G, Relacionamento interpessoal, mensura a capacidade do gestor de saber fazer e receber críticas, ter habilidades para dar feedbacks, conseguir ouvir as demandas do outro de modo empático e colocar-se à disposição para oferecer ajuda quando necessário, saber estabelecer comunicação assertiva e fazer e receber elogios. A pontuação média obtida pelos gestores nessa categoria foi de 3,11 , o que demonstra que eles se avaliaram positivamente quanto às habilidades de relacionamentos interpessoais para com seus liderados. Bolsoni-Silva et al. (2006) indicaram que pessoas com bons relacionamentos interpessoais são mais produtivas no trabalho e que o comportamento socialmente habilidoso contribui para a melhoria do clima organizacional. Essa categoria se mostra pertinente nesse contexto pois, conforme sinalizam De Rue e Myers (2014), as ações de liderar e ser seguido pelos liderados requerem competências de relacionamentos interpessoais em diferentes níveis, sejam esses macro, meso e/ou micro organizacional.
Os itens da categoria Cumprimento de metas e objetivos profissionais avaliam o repertório de habilidades sociais profissionais em termos de saber planejar rotinas e metas exequíveis de trabalho, distribuir tarefas e focar nos resultados profissionais esperados. Embora a pontuação tenha sido inferior a 3, percebeu-se que a média foi muito próximo a esse valor $(2,99)$, a partir do qual é possível supor que os resultados tendem a demonstrar uma percepção adequada por parte dos gestores quanto a suas capacidades de fazer a distribuição das tarefas do setor, em respeito aos interesses e habilidades dos membros de sua equipe. Tais dados se mostram relevantes uma vez que o cenário laboral atual lança desafios acentuados para os profissionais que atuam como gestores, pois eles precisam estar alinhados às novas exigências do contexto. Dessa forma, cada vez mais existe a necessidade de as organizações de trabalho captarem, desenvolverem e manterem profissionais capacitados para atuarem em níveis gerenciais (Faria, Lourencetti, Goulart Júnior, Cardoso, \& Camargo, 2014; Portz \& Amazarray, 2019; Rodrigues \& Faiad, 2019).

O terceiro conjunto de itens do IHSP-G, Respeito aos direitos e diferenças individuais, mensura a habilidade do gestor em conhecer as principais características de cada liderado, saber lidar com suas diferenças, respeitar os valores individuais, bem como ser ético nas relações e nas decisões de trabalho. Essa categoria foi a que apresentou maior média de respostas entre os gestores, informação altamente relevante no cenário atual de trabalho, já que, para além da valorização de seus subordinados, é necessário que o gestor tenha instrumental teórico para reconhecer e lidar com as diferenças de forma efetiva. Nessa mesma direção, atentar para as carências e necessidades individuais de cada trabalhador também parece ser importante aos modelos contemporâneos de gestão (Bryan et al., 2018; Gayed et al., 2018).

Também constatou-se média de resposta superior a 3 na categoria Comunicação organizacional, que se refere às habilidades de se promover e estimular diálogos profissionais inter e intrasetores, criar canais de comunicação efetivos no setor de trabalho, compartilhar informações e saber falar em público. A adequada e fluente comunicação é um fator fundamental para o desempenho satisfatório das equipes de trabalho. Certas estratégias de comunicação não dependem de recursos, e sim de criatividade dos gestores - como promover reuniões de esclarecimentos e orientações, 
treinamentos rápidos de capacitação, criar horários disponíveis para atendimentos individuais de seus liderados, disponibilizar informações por meio digital etc. Assim, gestores habilidosos em aspectos da comunicação podem ser vistos como importantes preditores de uma adequada formação de equipe de trabalho (Rodrigues et al., 2015).

Na categoria Resoluções de conflitos no trabalho é mensurada a capacidade dos gestores de saber ouvir e expressar a opinião em situações de conflito, saber identificar as situações geradoras de conflito e também o antecipar. Os resultados dessa categoria demonstraram que os gestores perceberam possuírem tais habilidades. Considerando a interdependência de tarefas entre setores, o bom relacionamento interpessoal é condição para o êxito do trabalho e, por isso, os gestores devem ficar atentos aos possíveis conflitos interpessoais entre os profissionais de seu setor e profissionais de outros setores, a fim de administrar conflitos existentes e, quando possível, antecipar-se a eles (Grubaugh \& Flynn, 2018; Hoffman \& Tadelis, 2021). Sobre a importância da presença de competências comportamentais no repertório dos profissionais na atualidade, Rodrigues et al. (2015), em um estudo de levantamento que buscou identificar as competências mais valorizadas nas organizações, identificaram que a resolução de problemas foi uma das competências destacadas.

Outra categoria com média de respostas superior a 3 foi a de Gestão do desempenho humano no trabalho, que mensurou os comportamentos dos gestores em relação a suas habilidades em avaliar cotidianamente o desempenho de seus liderados, favorecer a autonomia, identificar necessidades e carências do ambiente de trabalho e apontar para seus liderados as necessidades de capacitação e aprimoramento profissional. Considerando as constantes mudanças pelas quais os contextos de trabalho passam e a necessidade de implantar novas estratégias de ação, saber planejar ações de capacitação é condição para que os profissionais possam realizar as tarefas a contento e atingir metas e resultados esperados (Portz \& Amazarray, 2019; Rodrigues \& Faiad, 2019). Conforme destacaram Fleury e Fleury (2008), para que as competências dos trabalhadores sejam essenciais às organizações, elas devem estar associadas a um processo de aprendizagem que envolva inovação, novas descobertas e, especialmente, a capacitação dos indivíduos.
A categoria Gestão de pessoas do IHSP-G investiga o quanto os gestores possuem habilidades para criar um ambiente adequado à participação dos liderados nas decisões, estimular o trabalho em equipe, participar ativamente nos processos seletivos de novos trabalhadores e saber avaliar a necessidade de novos trabalhadores em seu setor. A menor média de resposta registrada neste estudo se deu nesta categoria, o que pode ser visto como um indicativo de dificuldade por parte dos gestores nesses quesitos. Pereira, Souza, Lucca e Iguti (2020) e Zanelli e Silva (2008) ressaltaram que os modelos de gestão a partir do século XXI passaram a requerer relações em que os gestores devem zelar pela saúde e pelo bem-estar das pessoas nos relacionamentos interpessoais. Esse tipo de comportamento, segundo os autores, está associado à maturidade dos gestores, na medida em que ele requer que os gestores reconheçam a importância da qualidade de vida no trabalho, bem como preocupem-se com a autoestima dos liderados, a fim de melhorar as condições ambientais e minimizar riscos psicossociais no trabalho.

$\mathrm{Na}$ última categoria avaliada, Lidar com o estresse e estressores profissionais, os itens mensuram as habilidades dos gestores em identificar os possíveis fatores geradores de tensão no contexto laboral e propor intervenções que caminhem na direção de um ambiente de trabalho mais saudável. Com base na média de respostas dos gestores, pode-se constatar que essa categoria não apresentou grandes problemas quanto aos comportamentos dos gestores avaliados, fato de relevância, pois a identificação de possíveis estressores no trabalho permite ao gestor se antecipar para que o fator seja eliminado e, na impossibilidade, preparar seus subordinados para lidarem com ele sem que traga prejuízos maiores para sua saúde física e mental e para a boa convivência no setor de trabalho (Bryan et al., 2018; Grubaugh \& Flynn, 2018).

De forma geral, na avaliação das médias das oito categorias do IHSP-G, observa-se, conforme a Tabela 1, que a análise que os gestores fizeram de si mesmos apontou para o respeito aos direitos e diferenças individuais como maior habilidade apresentada. Em contrapartida, gestão de pessoas foi o principal déficit encontrado. Silveira et al. (2016) explicam que um repertório habilidoso nas interações é importante para o convívio social. Esse repertório também é fundamental quando se trata de atuações nos complexos e instáveis contextos de trabalho. 
Por outro lado, o déficit de habilidades sociais também configura um fator de risco para o desenvolvimento de psicopatologias como a depressão (Hentrich et al., 2017; Segrin \& Taylor, 2007). Atualmente, doenças como depressão e ansiedade configuram-se como grandes causas de adoecimento no trabalho, de modo que estão relacionadas à diminuição da produtividade e do desempenho profissional, e afetam diretamente a qualidade de vida do indivíduo com essas morbidades. Fonseca e Carlotto (2011), nesse sentido, confirmam a depressão como um dos principais fatores que afetam a saúde do trabalhador, além de a relacionarem com o absenteísmo em ambientes de trabalho. Por isso, a presente pesquisa também mensurou os indicadores de ansiedade e depressão dos gestores avaliados. Essas informações estão presentes na Tabela 2.

Tabela 2

Indicadores de ansiedade e depressão nos gestores.

\begin{tabular}{lcc}
\hline & BAI & BDI \\
\hline Média & 27,64 & 5,81 \\
Pontuação máxima & 49,00 & 19,00 \\
Pontuação mínima & 2,00 & 0,00 \\
\hline
\end{tabular}

De acordo com a Tabela 2, a pontuação média obtida pelos gestores no Inventário de Ansiedade de Beck (BAI) e no Inventário de Depressão de Beck (BDI) foi de 27,64 e 5,81, respectivamente. De acordo com o manual dos testes, a interpretação dos resultados médios indica ansiedade moderada e ausência de depressão. Considerando as pontuações máxima e mínima no BAI, constata-se que há gestores com grau severo de ansiedade e outros, com grau mínimo. Já em relação aos escores do BDI, o maior grau de depressão encontrado no estudo aponta para o nível moderado. Há, portanto, maiores índices de ansiedade em comparação aos índices de depressão. Embora o gestor tenha uma série de responsabilidades para com seus liderados e com a organização, não é incomum encontrar gestores em grande sofrimento psíquico, devido à alta demanda de trabalho e à carga exacerbada de pressão que sofrem (Lourenção, 2018; Pires et al., 2018). Como forma de verificar possíveis associações entre as categorias do IHSP-G e os demais instrumentos aplicados, foi realizada a análise de correlação (Tabela 3).
Tabela 3

Correlações entre as categorias do IHSP-G e os demais instrumentos aplicados.

\begin{tabular}{|c|c|c|}
\hline CATEGORIAS IHS-G & BAI & BDI \\
\hline Relacionamento interpessoal & $-0,18$ & $-0,31$ \\
\hline $\begin{array}{l}\text { Cumprimento de metas e } \\
\text { objetivos profissionais }\end{array}$ & $-\mathbf{0 , 3 4} * *$ & $-0,47^{* *}$ \\
\hline $\begin{array}{l}\text { Respeito aos direitos e } \\
\text { diferenças individuais }\end{array}$ & $-0,39 * *$ & $-0,49^{* *}$ \\
\hline Comunicação organizacional & $-\mathbf{0 , 3 2 *}$ & $-\mathbf{0 , 3 4} *$ \\
\hline $\begin{array}{l}\text { Resoluções de conflitos no } \\
\text { trabalho }\end{array}$ & $-0,29$ & $-0,45^{* *}$ \\
\hline $\begin{array}{l}\text { Gestão do desempenho } \\
\text { humano no trabalho }\end{array}$ & $-0,06$ & $-0,30$ \\
\hline Gestão de pessoas & $-\mathbf{0 , 3 8} *$ & $-0,23$ \\
\hline $\begin{array}{l}\text { Lidar com o estresse e } \\
\text { estressores profissionais }\end{array}$ & $-0,24$ & $-0,38^{*}$ \\
\hline
\end{tabular}

Todas as correlações foram de sentido negativo, ou seja, quanto maior a presença de habilidades sociais profissionais, menores tendem a ser os indicadores de depressão e ansiedade nos gestores. Algumas correlações apresentaram significância estatística com os dois instrumentos (Cumprimento de metas e objetivos profissionais; Respeito aos direitos e diferenças individuais; Comunicação organizacional), outras, somente com o BDI (Resoluções de conflitos no trabalho; Lidar com o estresse e estressores profissionais) ou apenas com o BAI (Gestão de pessoas). Em que pese não ser possível pressupor uma relação causal entre os dados, é relevante formular a hipótese de que gestores habilidosos possuem menores indicadores de depressão e ansiedade (Hentrich et al., 2017; Lourenção, 2018; Pires et al., 2018).

Alguns estudos de habilidades sociais profissionais de gestores incluem aqueles realizados por Rimoldi, Silva, Goulart Júnior e Bolsoni-Silva (2011), que desenvolveram um programa de treino de habilidades sociais em 10 sessões voltado para os gestores da área da enfermagem de uma instituição hospitalar. Canêo e Santos (2010) também buscaram realizar Treino de Habilidades Sociais (THS), mas seus participantes eram os gestores de uma organização do segmento metalúrgico. Neste caso, os autores descrevem o treinamento feito por meio de exposições teóricas e role-playings voltados para conteúdo de habilidades sociais de comunicação, civilidade, assertividade, direito e cidadania, empáticas, de expressão de 
sentimento positivo, motivação, liderança, tomada de decisões e solução de problemas e conflitos. Reforça-se assim a compreensão de que as habilidades sociais profissionais podem ser aprendidas no próprio ambiente de trabalho mediante estratégias educativas bem planejadas e conduzidas.

\section{Considerações finais}

A presente pesquisa teve como objetivo analisar a percepção acerca do repertório de habilidades sociais profissionais de gestores, bem como seus indicadores de depressão e ansiedade. Os dados foram consistentes e atingiram os objetivos propostos. Porém, houve dificuldade na discussão teórica do construto e dos dados, pois, apesar do crescimento dos estudos em habilidades sociais, as publicações que abrangem esse repertório no contexto laboral ainda não são robustas. Frente a isso, os resultados apresentados neste artigo podem ser considerados de grande relevância para a literatura científica brasileira, não somente devido à importância das habilidades sociais profissionais de gestores, mas também pela escassez de investigações sobre a temática.

Nas publicações brasileiras, grande parte dos autores utilizaram instrumentos de habilidades sociais gerais para mensurar o construto em grupos de trabalhadores, comparando tais indicadores a outros do contexto laboral, tais como engajamento ou mesmo satisfação no trabalho. Entretanto, tais instrumentos não possuem, em muitos casos, estudos psicométricos com o referido público e, além disso, os itens não mensuram especificamente habilidades sociais profissionais. Esta pesquisa apresentou um instrumento (IHSP-G) que se encontra em processo de investigações quanto às qualidades psicométricas e que poderá, em curto prazo, ser considerado uma opção para a mensuração de habilidades sociais profissionais de gestores. É importante ressaltar as possibilidades de estudo a partir dessa ferramenta, pois ela considera os complexos e instáveis contextos organizacionais.

É válido destacar também que, embora seja uma coleta de dados apenas com gestores, o baixo número de participantes do presente estudo não permite a generalização de seus resultados, de modo que se recomenda sua replicação com uma amostragem maior. $\mathrm{O}$ aumento amostral também possibilitaria análises mais robustas, com criação de diferentes clusters, por exemplo, a fim de separar gestores que apresentarem baixos e elevados indicadores nos instrumentos de sintomatologias de ansiedade e depressão, e os comparar às frequências de comportamentos habilidosos relatados no IHSP-G.

Além disso, há uma tendência de as pessoas se considerarem socialmente habilidosas, o que é um fator a ser levado em consideração na análise dos resultados apresentados. Considerando que a cultura organizacional é determinante para os comportamentos das pessoas e para o desempenho das organizações, recomenda-se que os comportamentos socialmente habilidosos sejam ensinados e exercidos por meio de políticas, processos e práticas de gestão de pessoas, especialmente aqueles relacionados às práticas educativas no trabalho.

\section{Referências}

Argyle, M. (1980). The development of applied social psychology. In G. Gilmour \& S. Duck (Orgs.), The development of Social Psychology (pp. 1-22). Academic Press.

Argyle, M., Furnham, A., \& Graham, J. A. (1981). Social situations. Cambridge University Press.

Bolsoni-Silva, A. T., Del Prette, Z. A. P., Del Prette, G., Montagner, A. R., Bandeira, M., \& Del Prette, A. (2006). Habilidades sociais no Brasil: Uma análise dos estudos publicados em periódicos. In M. Bandeira, Z. A. P. Del Prette \& A. Del Prette (Orgs.), Estudos sobre habilidades sociais e relacionamento interpessoal (pp. 1-45). Casa do Psicólogo.

Bolsoni-Silva, A. T., \& Loureiro, S. R. (2016). O impacto das habilidades sociais para a depressão em estudantes universitários. Psicologia: Teoria e Pesquisa, 32(4), 1-8. https://doi.org/10.1590/0102.3772e324212

Bryan, B. T., Gayed, A., Milligan-Saville, J. S., Madan, I., Calvo, R. A., Glozier, N., \& Harvey, S. B. (2018). Managers' response to mental health issues among their staff. Occupational Medicine, 68(7), 464-468. https://doi.org/ $10.1093 /$ occmed/kqy103

Caballo, V. E. (2003). Manual de avaliação e treinamento em habilidades sociais. Santos.

Campos, J. R., Del Prette, A., \& Del Prette, Z. A. P. (2014). Depressão na adolescência: habilidades sociais e variáveis sociodemográficas como fatores de risco/proteção. Estudos e Pesquisas em Psicologia, 14(2), 408-428. 
Campos, J. R., Del Prette, A., \& Del Prette, Z. A. P. (2018). Relações entre depressão, habilidades sociais, sexo e nível socioeconômico em grandes amostras de adolescentes. Psicologia: Teoria e Pesquisa, 34, e3446. https://doi.org/10.1590/0102.3772e3446

Canêo, L. C., \& Santos, L. H. Z. (2010). Treinamento de Habilidades Sociais. In E. Goulart Júnior, L. C. Canêo \& M. C. F. Lunardelli (Orgs.), Experiências em Gestão de Pessoas nas Organizações de Trabalho (pp. 77-87). Joarte.

Cerutti, P. S., \&Wagner, M. F. (2014). Habilidades Sociais no Atendimento ao Público. Revista de Psicologia da IMED, 6(1), 40-46. https://doi.org/10.18256/2175-5027/psico-imed.v6n1p40-46

Cunha, J. A. (2001). Manual da versão em português das Escalas Beck. Casa do Psicólogo.

Cunha, L. F., \& Conceição, F. C. (2013). Habilidades sociais em militares de um esquadrão de helicópteros da marinha do Brasil. Revista Conexão Sipaer, 4(2), 69-77.

Del Prette, Z. A. P., \& Del Prette, A. (2001). Psicologia das relações interpessoais: Vivências para o trabalho em grupo. Vozes.

Del Prette, Z. A. P., \& Del Prette, A. (2002). Transtornos psicológicos e habilidades sociais. In H. J. Guilhardi, M. B. B. P. Madi, P. P. Queiroz \& M. C. Scoz (Orgs.), Sobre comportamento e cognição. Contribuições para a construção da teoria do comportamento (pp. 377-386). ESETec.

De Rue, D. S, \& Myers, C. G. (2014). Leardership development: A review and agenda for future research. In D. V. Day (Ed.), The Oxford Handbook of Leardership and Organizations (pp. 832-855). Oxford University Press.

Falcone, E. O. (2002). Contribuições para o treinamento de habilidades de interação. In H. J. Guilhardi, M. B. B. P. Madi, P. P. Queiroz \& M. C. Scoz (Orgs.), Sobre comportamento e cognições. Contribuições para a construção da teoria do comportamento (pp. 91-104). ESETec.

Faria, L. E., Lourencetti, L. A., Goulart Júnior, E., Cardoso, H. F., \& Camargo, M. L. (2014). Organização Pública e Cultura organizacional: necessidades de mudanças e revigoramento em direção à eficiência [Trabalho apresentado]. Anais do XXI Simpósio de Engenharia de Produção, Bauru, São Paulo, Brasil. http://www.simpep.feb.unesp.br/anais_simpep.php?e=9

Fleury, A., \& Fleury, M. T. L. (2008). Estratégias empresariais e formação de competências: um quebra-cabeças caleidoscópio da indústria brasileira (3a ed.). Atlas.

Fonseca, R. M. C., \& Carlotto, M. S. (2011). Saúde mental e afastamento do trabalho em servidores do Judiciário do Estado do Rio Grande do Sul. Psicologia e Pesquisa, 5(2), 117-125.

Gayed, A., Milligan-Saville, J. S., Nicholas, J., Bryan, B. T., LaMontagne, A. D., Milner, A., Madan, I., Calvo, R. A., Christensen, H., Mykletun, A., Glozier, N., \& Harvey, S. B. (2018). Effectiveness of training workplace managers to understand and support the mental health needs of employees: a systematic review and meta-analysis. Occupational and Environmental Medicine, 75, 462-470. https://doi.org/10.1136/oemed-2017-104789

Goulart Júnior, E., Camargo, M. L., \& Moreira, M. C. (2019). Habilidades sociais profissionais: produção científica nacional e relevância do tema para a saúde dos trabalhadores. Revista de Psicologia, 10(2), 41-50. http://periodicos.ufc.br/psicologiaufc/article/view/39967

Goulart Júnior, E., Cardoso, H. F., Bolsoni-Silva, A. T., \& Silveira, A. M. (2017). Inventário de Habilidades Sociais Profissionais [Manual técnico não publicado]. Universidade Estadual Paulista.

Grubaugh, M. L., \& Flynn, L. (2018). Relationships Among Nurse Manager Leadership Skills, Conflict Management, and Unit Teamwork. The Journal of Nursing Administration, 48(7/8), 383-388. https://doi.org/10.1097/ NNA.0000000000000633

Hentrich, S., Zimber, A., Sosnowsky-Waschek, N., Gregersen, S., \& Petermann, F. (2017). The Role of Core SelfEvaluations in Explaining Depression and Work Engagement among Managers. Current Psychology, 36, $516-529$. https://doi.org/10.1007/s12144-016-9439-x

Hoffman, M., \& Tadelis, S. (2021). People Management Skills, Employee Attrition, and Manager Rewards: An Empirical Analysis. Journal of Political Economy, 129(1), 243-285. https://doi.org/10.1086/711409

Lourenção, L. G. (2018). Qualidade de vida, engagement, ansiedade e depressão entre gestores de Unidades da Atenção Primária à Saúde. Revista Portuguesa de Enfermagem de Saúde Mental, 20, 58-64. https://doi.org/10.19131/rpesm.0227

Pereira, A. C. L., Souza, H. A., Lucca, S. R. D., \& Iguti, A. M. (2020). Fatores de riscos psicossociais no trabalho: limitações para uma abordagem integral da saúde mental relacionada ao trabalho. Revista Brasileira de Saúde Ocupacional, 45, 1-9. https://doi.org/10.1590/2317-6369000035118

Pires, F. B. C., Lacerda, S. S., Balardin, J. B., Portes, B., Tobo, P. R., Barrichello, C. R. C., Amaro Jr., E., \& Kozasa, E. H. (2018). Self-compassion is associated with less stress and depression and greater attention and brain response to affective stimuli in women managers. BMC Women's Health, 18(195), 1-7. https://doi.org/10.1186/ s12905-018-0685-y 
Portz, R. M., \& Amazarray, M. R. (2019). Transtornos mentais comuns e fatores associados em trabalhadores bancários do Rio Grande do Sul, Brasil. Revista Psicologia: Organizações e Trabalho, 19(1), 515-522. https:// doi.org/10.17652/rpot/2019.1.13326

Rodrigues, C. M. L., \& Faiad, C. (2019). Pesquisa sobre riscos psicossociais no trabalho: estudo bibliométrico da produção nacional de 2008 a 2017. Revista Psicologia: Organizações e Trabalho, 19(1), 571-579. https:// doi.org/10.17652/rpot/2019.1.15424

Rodrigues, M. S., Araújo, E. A. S., Ribeiro, M. J. F. X., \& Rubio, J. M. L. (2015). Habilidades interpessoais nas competências gerenciais e de liderança. In Z. A. Del Prette, A. B. Soares, C. S. Pereira-Guizzo, M. F. Wagner \&V. B. R. Leme (Orgs.), Habilidades Sociais: Diálogos e intercâmbios sobre pesquisa e prática (pp. 462-496). Sinopsys.

Rimoldi, L. P., Silva, V.V. P., Goulart Júnior, E., \& Bolsoni-Silva, A. T. (2011). Programa de Treinamento de Habilidades Sociais Para Gestores de Enfermagem de uma Instituição Hospitalar. In E. Goulart Júnior \& L. C. Canêo (Orgs.), Aprendizagens iniciais na área de Psicologia Organizacional e do Trabalho (pp. 134-144). Joarte.

Segrin, C., \& Rynes, K. N. (2009). The mediating role of positive relations with others in associations between depressive symptoms, social skills, and perceived stress. Journal of Research in Personality, 43(1), 962-971. https://doi.org/10.1016/j.jrp.2009.05.012

Segrin, C., \& Taylor, M. (2007). Positive interpersonal relationships mediate the association between social skills and psychological well-being. Personality and Individual Differences, 43(1), 637-646. https://doi.org/10.1016/ j.paid.2007.01.017

Silveira, A. M., Goulart Júnior, E., Cardoso, H. F., \& Bolsoni-Silva, A. T. (2016). Identificação de Habilidades Sociais Profissionais em Líderes que atuam em Unidades Acadêmicas de uma IES-Pública. In E. Goulart Júnior, H. F. Cardoso, M. R. Feijó, M. L. Camargo \& D. C. Campos (Orgs.), Psicologia Organizacional e do Trabalho e Orientação Profissional na formação do psicólogo (pp. 57-71). Letraria.

Zanelli, J. C., \& Silva, N. (2008). Interação Humana e Gestão: a construção psicossocial das organizações de trabalho. Casa do Psicólogo.

\section{Edward Goulart Júnior}

Psicólogo e Doutor em Psicologia pela Pontifícia Universidade Católica de Campinas (PUC-Campinas), Campinas - SP. Brasil. Professor Assistente Doutor do Departamento de Psicologia da Universidade Estadual Paulista Júlio de Mesquita Filho (Unesp), Bauru - SP. Brasil.

E-mail: edward.goulart@unesp.br

(1) https:// orcid.org/0000-0002-4923-433X

\section{Hugo Ferrari Cardoso}

Psicólogo e Doutor em Psicologia pela Universidade São Francisco (USF), Bragança Paulista - SP. Brasil. Professor Assistente Doutor do Departamento de Psicologia da Universidade Estadual Paulista Júlio de Mesquita Filho (Unesp), Bauru - SP. Brasil.

E-mail: hugo.cardoso@unesp.br

(1) https://orcid.org/0000-0003-1960-2936

\section{Thaís Almeida Alves}

Graduada em Psicologia pela Universidade Estadual Paulista Júlio de Mesquita Filho (Unesp), Bauru - SP. Brasil.

E-mail: thais_almeida_alves@hotmail.com

(1) https://orcid.org/0000-0002-4569-6297

\section{Aline de Marco da Silveira}

Universidade Estadual Paulista Júlio de Mesquita Filho

Graduada em Psicologia pela Universidade Estadual Paulista Júlio de Mesquita Filho (Unesp), Bauru - SP. Brasil.

E-mail: alinedmarco@gmail.com

(1) https://orcid.org/0000-0002-0286-780X 
Endereço para envio de correspondência:

Universidade Estadual Paulista, Campus de Bauru, Departamento de Psicologia da Faculdade de Ciências. Avenida Engenheiro Luiz Edmundo Carrijo Coube, 14-01, Vargem Limpa. CEP: 17033-360. Bauru - SP. Brasil.

Recebido 27/03/2019

Aceito 22/06/2021

Received 03/27/2019

Approved 06/22/2021

Recibido 27/03/2019

Aceptado 22/06/2021

Como citar: Goulart Júnior, E., Cardoso, H. F., Alves, T. A., \& Silveira, A. M. (2021). Habilidades Sociais Profissionais e Indicadores de Ansiedade e Depressão em Gestores. Psicologia: Ciência e Profissão, 41, 1-12. https://doi. org/10.1590/1982-3703003221850

How to cite: Goulart Júnior, E., Cardoso, H. F., Alves, T. A., \& Silveira, A. M. (2021). Professional Social Skills and Indicators of Anxiety and Depression in Managers. Psicologia: Ciência e Profissão, 41, 1-12. https://doi.org/ 10.1590/1982-3703003221850

Cómo citar: Goulart Júnior, E., Cardoso, H. F., Alves, T. A., \& Silveira, A. M. (2021). Habilidades Sociales Profesionales e Indicadores de Ansiedad y Depresión en Gestores. Psicologia: Ciência e Profissão, 41, 1-12. https://doi.org/10.1590/1982-3703003221850 